\title{
Quantum Correlations of Light from a Room-Temperature Mechanical Oscillator
}

\author{
V. Sudhir, R. Schilling, S. A. Fedorov, H. Schütz, D. J. Wilson, and T. J. Kippenberg* \\ Institute of Physics, École Polytechnique Fédérale de Lausanne, Lausanne 1015, Switzerland \\ (Received 23 May 2017; revised manuscript received 7 August 2017; published 26 September 2017) \\ When an optical field is reflected from a compliant mirror, its intensity and phase become quantum- \\ correlated due to radiation pressure. These correlations form a valuable resource: the mirror may be viewed \\ as an effective Kerr medium generating squeezed states of light, or the correlations may be used to erase \\ backaction from an interferometric measurement of the mirror's position. To date, optomechanical quantum \\ correlations have been observed in only a handful of cryogenic experiments, owing to the challenge of \\ distilling them from thermomechanical noise. Accessing them at room temperature, however, would \\ significantly extend their practical impact, with applications ranging from gravitational wave detection to \\ chip-scale accelerometry. Here, we observe broadband quantum correlations developed in an optical field \\ due to its interaction with a room-temperature nanomechanical oscillator, taking advantage of its high- \\ cooperativity near-field coupling to an optical microcavity. The correlations manifest as a reduction in the \\ fluctuations of a rotated quadrature of the field, in a frequency window spanning more than an octave below \\ mechanical resonance. This is due to coherent cancellation of the two sources of quantum noise \\ contaminating the measured quadrature-backaction and imprecision. Supplanting the backaction force \\ with an off-resonant test force, we demonstrate the working principle behind a quantum-enhanced \\ "variational" force measurement.
}

DOI: 10.1103/PhysRevX.7.031055

\section{INTRODUCTION}

The radiation pressure interaction of light with mechanical oscillators has been the subject of intense theoretical research in the gravitational wave community [1-3], leading, for example, to an understanding of the quantum limits of interferometric position measurements. An important insight that could potentially help evade this limit is that the two noise sources that enforce it-quantum (measurement) backaction and imprecision-are, in general, correlated [4]. From the perspective of the light in the interferometer, quantum fluctuations in its amplitude quadrature drive the oscillator leading to quantum backaction, and the driven motion is imprinted onto the phase quadrature. Ultimately, this leads to correlations between the quantum fluctuations of the amplitude and phase quadratures, i.e., quantum correlations. Correlations thus established form a valuable resource: the optomechanical system may be viewed as an effective Kerr medium generating squeezed states of the optical field $[5,6]$, or the correlations can be directly employed for backaction cancellation [7-10]. Indeed, the ability to utilize quantum correlations generated in situ is conceptually identical to injection of squeezed light [9], while circumventing the challenge of realizing a near-unity

\footnotetext{
*tobias.kippenberg@epfl.ch
}

Published by the American Physical Society under the terms of the Creative Commons Attribution 4.0 International license. Further distribution of this work must maintain attribution to the author(s) and the published article's title, journal citation, and DOI.
Subject Areas: Mechanics, Optics, Quantum Physics

coupling efficiency between the squeezed light source and the interferometer [11]. The burden of quantum efficiency in this case is shifted to the detector, a problem that is largely solved [12]. Thus, a room-temperature interferometer capable of harnessing in situ correlations is a platform that may help extend the practical reach of quantum optics, with applications ranging from gravitational wave detection to chip-scale accelerometry.

In practice, owing to the weakness of the radiation pressure force, optomechanical quantum correlations are typically obscured by thermal motion. Only in recent years has this challenge been broached, by the development of cavity optomechanical systems [13], which combine an engineered high- $Q$, cryogenically cooled micromechanical oscillator with a high-finesse optical (or microwave) cavity. In such systems, it is possible to realize a regime in which the motion of the oscillator is dominated-or nearly so-by quantum backaction [14-16]. This has enabled studies of various effects related to optomechanical quantum correlations, such as ponderomotive squeezing [17-21] and motional sideband asymmetry (using autonomous [22-25] or measurementbased [20] feedback to cool the mechanical oscillator). Accessing this regime at room temperature is difficult, as the optical powers necessary to overwhelm thermal forces with backaction are typically accompanied by dynamic instabilities [26]. Various cross-correlation techniques have been proposed to relax these requirements and allow observation of quantum correlations near mechanical resonance $[27,28]$. In a recent demonstration [29], such a technique was used in the regime of large measurement imprecision to unearth quantum correlations from beneath $60 \mathrm{~dB}$ of 
thermal noise. However, the generation of broadband quantum-noise-induced optomechanical correlations, that could simultaneously ameliorate the limits posed by imprecision and backaction on room-temperature interferometers [7-9,30,31], remains an outstanding challenge.

Here, we observe quantum correlations of light generated by its interaction with a room-temperature nanomechanical oscillator coupled to a high-finesse optical microcavity and discuss how to use these correlations to realize a quantumenhanced mechanical force sensor. The large bandwidth of the cavity and the exceptionally high $Q /$ mass of the oscillator allows us to operate in a novel regime where the magnitude of quantum correlations is comparable to both sources of quantum noise-imprecision and backaction-at Fourier frequencies detuned as much as an octave from mechanical resonance. In a suitably chosen field quadrature, correlations manifest as a reduction or enhancement in the fluctuation spectrum at Fourier frequencies detuned from mechanical resonance. Though the fluctuations are contaminated by thermal noise and do not drop below the vacuum level - the condition for ponderomotive squeezing - their reduction provides a metrological advantage, since its origin is a coherent cancellation of quantum noises. Counterintuitively, as a result of this coherence, we observe a $10 \%$ noise reduction despite the fact that backaction is $20 \mathrm{~dB}$ smaller, in equivalent phonon units $\left(n_{\mathrm{QBA}}\right)$, than thermal noise $\left(n_{\text {th }} \approx k_{B} T / \hbar \Omega_{m} \approx 10^{6}\right)$. Indeed, at optimal Fourier frequencies, the fractional noise reduction scales as $\sqrt{n_{\mathrm{QBA}} / n_{\mathrm{th}}}$, distinguishing it from classical-noise-induced correlations, and enabling an enhanced estimate of the quantum backaction force relative to standard "calorimetric" measurements [14-16]. We conclude by showing how quantum correlations can be used to improve the signalto-noise ratio of an off-resonant test force.

\section{CONCEPT OF THE EXPERIMENT}

Accessing the above physics requires the ability to resolve backaction-driven motion far from mechanical resonance, a regime traditionally studied for gravitational wave detectors, but only recently accessed experimentally, using high-cooperativity cryogenic microcavity optomechanical systems operating deep in the so-called bad cavity limit $[15,20]$. Our system is optimized for achieving this regime at room temperature and is detailed elsewhere [32]. As shown in Fig. 1, it consists of a $\mathrm{Si}_{3} \mathrm{~N}_{4}$ nanomechanical beam coupled dispersively to a whispering gallery mode of a silica microdisk. The beam has an exceptionally high room-temperature $Q /$ mass factor due to its high stress and quasi-1D geometry. To enhance its optomechanical coupling, it is suspended $\approx 50 \mathrm{~nm}$ from the surface of the disk and profiled to increase its overlap with the optical mode [see Supplemental Material (SM) [33]]. This results in a vacuum optomechanical coupling rate of $g_{0} \approx 2 \pi \times 60 \mathrm{kHz}$ for the fundamental, $\Omega_{m}=2 \pi \times 3.4 \mathrm{MHz}$ flexural beam mode - a threefold increase over previous implementations

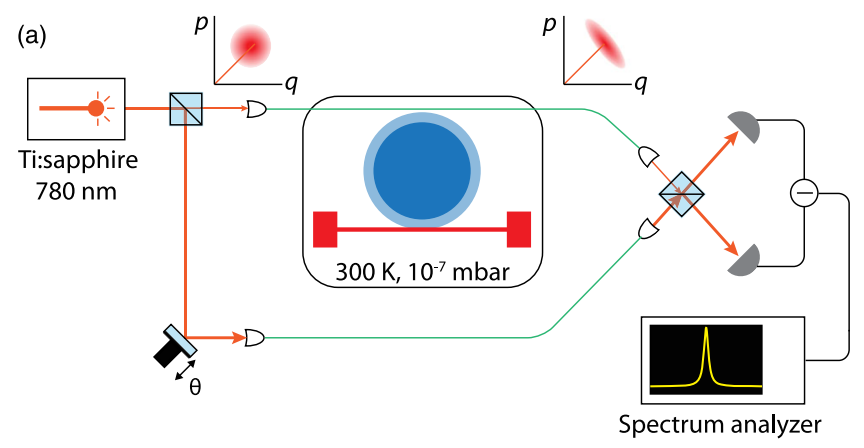

(b)

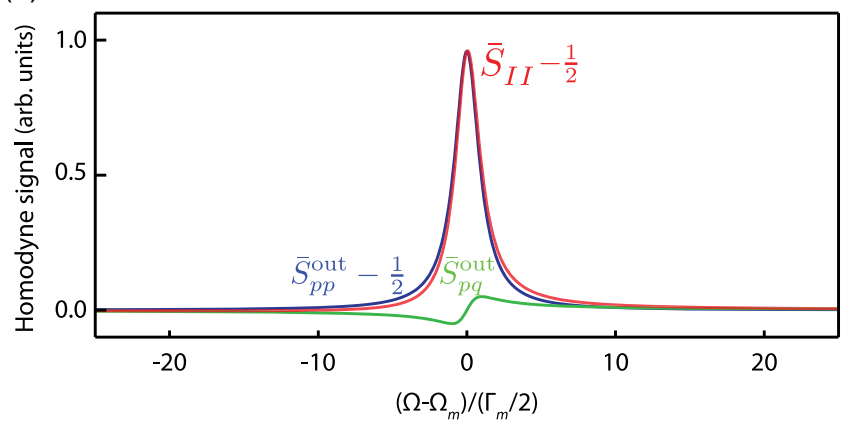

FIG. 1. Optomechanical quantum correlations. (a) Schematic of the experiment: The optomechanical system is formed by a $\mathrm{Si}_{3} \mathrm{~N}_{4}$ nanobeam oscillator (red) evanescently coupled to $\mathrm{SiO}_{2}$ microdisk cavity (blue). Both are maintained at room temperature $(T \approx 300 \mathrm{~K})$ in a low-pressure $\left(\approx 10^{-7} \mathrm{mbar}\right)$ vacuum chamber. The cavity is probed on resonance with 780-nm light from a Ti: sapphire laser. The transmitted field is read out with a homodyne detector with variable local oscillator phase $\theta$. Amplitude and phase fluctuations of the light field are correlated after passing through the cavity, represented as squashing in a phase space cartoon. (b) Model of the homodyne photocurrent spectrum [normalized to vacuum noise, Eq. (9)] for detection near the amplitude quadrature $\left(\theta \approx 0^{\circ}\right)$. The signal (red line) has a symmetric part (blue line) due to physical motion and an asymmetric part (green line) due to quantum correlations.

of the device $[15,20]$. In conjunction with the high roomtemperature mechanical quality factor, $Q_{m} \approx 3 \times 10^{5}$ (giving a damping rate of $\Gamma_{m}=\Omega_{m} / Q_{m} \approx 2 \pi \times 12 \mathrm{~Hz}$ ), and a critically coupled cavity decay rate of $\kappa \approx 2 \pi \times 4.5 \mathrm{GHz}$, a near-unity single photon cooperativity of $C_{0}=4 g_{0}^{2} / \kappa \Gamma_{m} \approx$ 0.27 is attained. Importantly, the system operates in the broadband regime, i.e., $\kappa \gg \Omega_{m}$.

In the experiment [outlined in Fig. 1(a)], the optomechanical device is placed in a high-vacuum chamber and probed on resonance using a Ti:sapphire laser. The motion of the oscillator, characterized by the Fourier transform of its displacement fluctuation $\delta x[\Omega]$, is imprinted on the transmitted phase quadrature as (see SM [33])

$$
\delta p_{\text {out }}[\Omega]=-\delta p_{\text {in }}[\Omega]+\sqrt{2 C \Gamma_{m}} \frac{\delta x[\Omega]}{x_{\mathrm{zp}}},
$$

where $p_{\text {in }}$ is the phase quadrature of the input field, $x_{\mathrm{zp}}=$ $\sqrt{\hbar / 2 m \Omega_{m}}$ is the zero-point motion of the oscillator, and 
$C=C_{0} n_{c}$ is the multiphoton cooperativity for the mean intracavity photon number $n_{c}$. The displacement has components from the ambient thermal environment, and quantum backaction, viz.,

$$
\delta x[\Omega]=\delta x_{\mathrm{th}}[\Omega]+\delta x_{\mathrm{QBA}}[\Omega] .
$$

The thermal motion,

$$
\delta x_{\mathrm{th}}[\Omega]=2 x_{\mathrm{zp}} \chi[\Omega] \sqrt{\left(n_{\mathrm{th}}+\frac{1}{2}\right) / \Gamma_{m}} \delta \xi[\Omega],
$$

is due to a Langevin force of intensity proportional to the average thermal occupation $n_{\mathrm{th}}$, and fluctuating as a white noise $\delta \xi$, characterized by

$$
\left\langle\delta \xi(t) \delta \xi\left(t^{\prime}\right)\right\rangle=\delta\left(t-t^{\prime}\right) .
$$

The force fluctuations are transduced into displacement fluctuations via the dimensionless susceptibility of the oscillator,

$$
\chi[\Omega] \equiv \frac{\Omega_{m} \Gamma_{m}}{\Omega_{m}^{2}-\Omega^{2}-i \Omega \Gamma_{m}}=\left(m \Omega_{m} \Gamma_{m}\right) \chi_{x}[\Omega],
$$

where $\chi_{x}$ is the conventional susceptibility. The backactiondriven motion,

$$
\delta x_{\mathrm{QBA}}[\Omega]=2 x_{\mathrm{zp}} \chi[\Omega] \sqrt{2 n_{\mathrm{QBA}} / \Gamma_{m}} \delta q_{\mathrm{in}}[\Omega],
$$

arises from quantum fluctuations in the amplitude quadrature of the input field $\delta q_{\text {in }}$, characterized by [34]

$$
\left\langle\delta q_{\text {in }}(t) \delta q_{\text {in }}\left(t^{\prime}\right)\right\rangle=\frac{1}{2} \delta\left(t-t^{\prime}\right),
$$

and leads to an additional phonon occupation, $n_{\mathrm{QBA}}=C$.

Because $\delta q_{\text {in }}$ is written onto the output optical phase vis-à-vis backaction, the amplitude and phase quantum fluctuations of the output field are correlated. The magnitude of these quantum correlations is characterized by the symmetrized cross-correlation spectrum (see SM [33]),

$$
\begin{aligned}
\bar{S}_{p q}^{\text {out }}[\Omega] & \equiv \int\left\langle\frac{1}{2}\left\{\delta p_{\text {out }}(t), \delta q_{\text {out }}(0)\right\}\right\rangle e^{i \Omega t} d t \\
& =2 \eta n_{\mathrm{QBA}} \operatorname{Re} \chi[\Omega],
\end{aligned}
$$

which is proportional to the backaction occupation, and the overall detection efficiency $\eta$. The correlation changes sign across the mechanical resonance frequency because of the $180^{\circ}$ phase change in the response of the oscillator to the quantum backaction force.

Phase-amplitude correlations can be experimentally accessed by measuring a linear superposition of both the amplitude and phase of the transmitted field. Proper choice of the superposition, exemplified by a homodyne detector, allows for a quantum-limited measurement of both quadratures. Specifically, the transmitted field is interfered at a beam splitter with a strong local oscillator field at a fixed phase offset $\theta$, followed by balanced detection of the two outputs of the beam splitter. In this case, the photocurrent $I_{\theta}$ is proportional to the rotated field quadrature, $\delta q_{\theta}=\delta q \cos \theta+\delta p \sin \theta$. Its spectrum therefore contains contributions due to amplitude or phase vacuum noise, oscillator motion, and quantum correlations, viz.,

$\bar{S}_{I I}^{\theta}[\Omega] \propto \cos ^{2} \theta \bar{S}_{q q}^{\text {out }}[\Omega]+\sin ^{2} \theta \bar{S}_{p p}^{\text {out }}[\Omega]+\sin (2 \theta) \bar{S}_{p q}^{\text {out }}[\Omega]$,

where

$$
\begin{aligned}
& \bar{S}_{q q}^{\text {out }}[\Omega]=\frac{1}{2}, \\
& \bar{S}_{p p}^{\text {out }}[\Omega]=\frac{1}{2}+8 \eta C|\chi[\Omega]|^{2}\left(n_{\text {tot }}+\frac{1}{2}\right)
\end{aligned}
$$

are, respectively, the transmitted amplitude quadrature spectrum (containing a copy of the incident vacuum fluctuations) and the transmitted phase quadrature spectrum (carrying, in addition, the total motion of the oscillator transduced via the optomechanical interaction). Here, $n_{\text {tot }}=n_{\text {th }}+n_{\mathrm{QBA}}$ is the phonon occupation of the oscillator due to the combined effect of the thermal and backaction forces.

The homodyne photocurrent spectrum [in Eq. (9)], expressed in terms of the mechanical response,

$$
\begin{gathered}
\bar{S}_{I I}^{\theta}[\Omega] \propto 1+16 \eta C|\chi[\Omega]|^{2}\left(n_{\mathrm{tot}}+\frac{1}{2}\right) \sin ^{2} \theta \\
+4 \eta C \operatorname{Re} \chi[\Omega] \sin 2 \theta
\end{gathered}
$$

consists of a measurement imprecision due to vacuum fluctuations of the detected quadrature, a component due to the motion of the oscillator diminished by a $\sin ^{2} \theta$ factor, and a component due to correlations between the imprecision and the quantum backaction $(\propto \sin 2 \theta)$. By exploiting its different dependence on the homodyne angle and Fourier frequency, the correlation term may be detected despite the large thermal motion of the oscillator at room temperature. Specifically, near the amplitude quadrature $\left(\theta=0^{\circ}\right)$ and at Fourier frequencies detuned from mechanical resonance $\left(\left|\Omega-\Omega_{m}\right| \gtrsim \Gamma_{m}\right)$, the contribution of thermal and backaction forces is suppressed relative to the correlation term. Closer inspection shows that a necessary requirement for the correlation term to dominate Eq. (11) is that the backaction force dominates the thermal force: $n_{\mathrm{QBA}} \gtrsim n_{\mathrm{th}}$.

The large thermal occupation of room-temperature mechanical oscillators makes it technically challenging to 
achieve $n_{\mathrm{QBA}}>n_{\mathrm{th}}$. Nevertheless, even when $n_{\mathrm{QBA}} \ll n_{\mathrm{th}}$, the signature of quantum correlations can still be discerned in the homodyne photocurrent spectrum at frequencies far detuned from mechanical resonance (alternate detection techniques have been demonstrated [20,23], and proposed [35], to detect backaction-induced quantum correlations on mechanical resonance). To wit, for detunings $\delta \equiv \Omega-\Omega_{m}$, which are larger than $\Gamma_{m}$, the homodyne photocurrent spectrum takes on a signature antisymmetry with respect to both $\delta$ and $\theta[27,28]$ :

$$
\begin{aligned}
& \bar{S}_{I I}^{\theta}\left[\Omega_{m}+\delta\right]_{|\delta| \gg \Gamma_{m}} \\
& \quad \approx 1+4 \eta C\left(\frac{\Gamma_{m}}{\delta}\right)^{2} \sin ^{2} \theta\left(n_{\mathrm{th}}+n_{\mathrm{QBA}}-\frac{\delta}{\Gamma_{m}} \cot \theta\right) .
\end{aligned}
$$

Note that such an antisymmetry can also arise from quantum correlations established by injecting squeezed light into the optical cavity [36] (or, indeed, classical correlations established by injecting a laser field with classical amplitude fluctuations [37,38]). Figure 1(b) shows a model of the homodyne photocurrent spectrum for a quadrature close to the amplitude (i.e., $\theta \approx 0^{\circ}$ ): the red trace represents the asymmetric spectrum observed at sufficiently large optical powers and the blue and green traces represent contributions due to thermal motion and quantum correlations, respectively.

\section{OBSERVATION OF QUANTUM CORRELATIONS}

In the following, we discuss homodyne measurements of a rotated quadrature of the field transmitted through our room-temperature nanobeam-microdisk optomechanical system, with powers sufficient to resolve the asymmetry due to quantum correlations. In order to mitigate optomechanical instabilities, active radiation pressure feedback is used to damp the mechanical mode [15,39]. For this purpose we employ an auxiliary 850-nm laser side-locked to an independent cavity mode (see SM [33]). Cold damping of this sort changes the mechanical susceptibility within the feedback bandwidth $(1 \mathrm{kHz}$ in this instance); nevertheless, the total decoherence rate, and the ratio $n_{\mathrm{QBA}} / n_{\text {th }}$, remains unchanged. Figure 2(a) shows the sensitivity of the homodyne interferometer as a function of the local oscillator phase $\theta$. By operating with a modest input power of $280 \mu \mathrm{W}$, we measure thermal motion of the oscillator with an (phonon-equivalent) imprecision, $n_{\mathrm{imp}}=$ $(16 \eta C)^{-1} \approx 4 \times 10^{-5}$, that is approximately $50 \mathrm{~dB}$ below that at the standard quantum limit (SQL) (i.e., $n_{\text {imp }}=1 / 4$ ) while operating on phase quadrature $\left(\theta=90^{\circ}\right)$. As the local oscillator phase is swept towards the amplitude quadrature $\left(|\theta| \rightarrow 0^{\circ}\right)$, the apparent thermal motion is
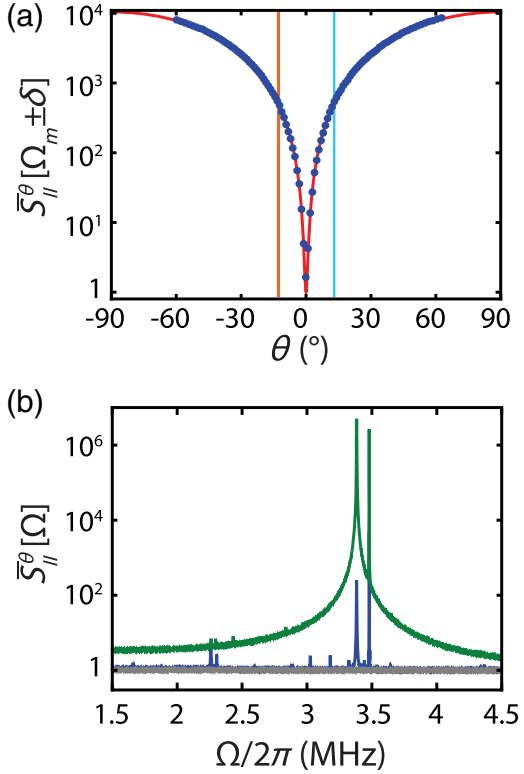

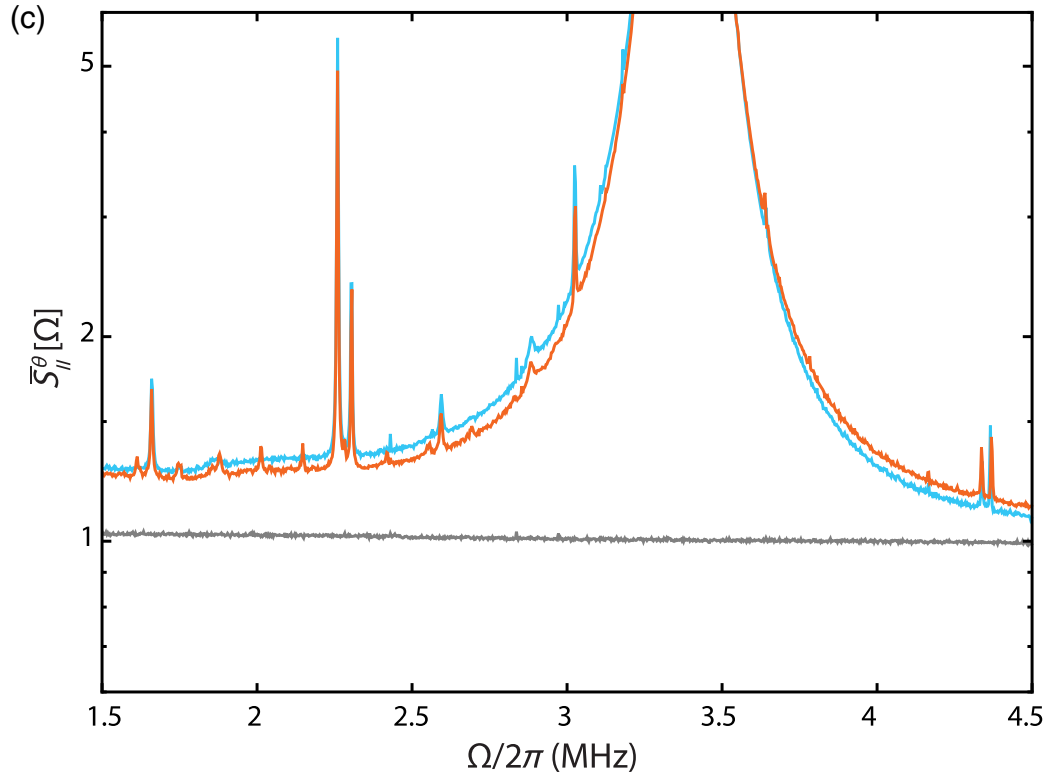

FIG. 2. Asymmetry in homodyne spectrum. (a) Resonant magnitude of the photocurrent signal $\bar{S}_{I I}^{\theta}\left[\Omega_{m}\right]$ (normalized to shot noise) as a function of the homodyne angle $\theta$. Blue points are measurements. Red line is a fit to Eq. (11). 40-dB suppression of the signal is achieved on the amplitude quadrature, limited by residual fluctuations in the homodyne angle $\left(\theta_{\text {rms }}<0.57^{\circ}\right)$. (b) Example spectra taken near the phase (green) and amplitude (blue) quadratures. Also plotted is the background with the meter laser blocked (gray), dominated by LO shot noise (detector electronic noise is $10 \mathrm{~dB}$ below shot noise). For all measurements, feedback is used to stabilize the mechanical mode, as discussed in the main text. Note that the sharp peak at $3.5 \mathrm{MHz}$ is due to thermal motion of the fundamental inplane beam mode. (c) Magnified image of the spectrum at two quadratures, $\theta= \pm 13^{\circ}$, highlighted with vertical lines in (a) (blue is $+13^{\circ}$, yellow is $-13^{\circ}$ ). The $\sim 10 \%$ asymmetry between the two spectra at Fourier frequency detunings away from mechanical resonance $\left(\Omega_{m} \approx 2 \pi \times 3.5 \mathrm{MHz}\right)$ arises due to quantum correlations [last term in Eq. (11)]. Larger asymmetry is observed at Fourier frequencies farther from mechanical resonance, as predicted by Eq. (12). The spectra are measured at an injected power of $P_{\text {in }}=280 \mu \mathrm{W}$. 
suppressed by about $40 \mathrm{~dB}$. Figure 2(b) shows example photocurrent spectra measured close to the phase (green) and amplitude (blue) quadratures; the gray trace shows shot noise of the homodyne detector, recorded by blocking the meter field. Excess amplitude noise in the output field is measured to be $\approx 1 \%$ of shot noise for the largest powers used in our experiments $(P \approx 280 \mu \mathrm{W})$, which we attribute to thermally driven fluctuations of the tapered fiber (see SM [33]).

In order to visualize the asymmetry in the photocurrent spectra as predicted by Eq. (12), we compare two spectra at homodyne angles symmetric about the amplitude quadrature, indicated by the blue (at angle $+\theta$ ) and orange (at angle $-\theta$ ) vertical lines in Fig. 2(a). The corresponding spectra are shown in Fig. 2(c). An asymmetry of approximately $10 \%$ with respect to Fourier frequency is observed at detunings $\delta \gtrsim 2 \pi \times 1 \mathrm{kHz}$, consistent with the theoretically predicted effect due to quantum correlations. Qualitatively, the observed asymmetry over a broad range of frequencies-more than a $\mathrm{MHz}$ on either side of resonance-indicates that the magnitude of quantumnoise-induced correlations is comparable to all sources of quantum noise at these frequencies.

The asymmetry in the observed spectrum [red in Fig. 2(c)] traces its root to the detuning dependence of quantum correlations [green trace in Fig. 1(b) and third term in Eq. (12)]. In order to systematically investigate this asymmetry, we consider the ratio of the homodyne photocurrents at frequency offsets at $\pm \delta$ from mechanical resonance:

$$
R_{\theta} \equiv \frac{\bar{S}_{I I}^{\theta}\left[\Omega_{m}+\delta\right]}{\bar{S}_{I I}^{\theta}\left[\Omega_{m}-\delta\right]} .
$$

Following Eq. (12),

$R_{\theta}=\frac{1+4 \eta C n_{\mathrm{tot}}\left(\Gamma_{m} \sin \theta / \delta\right)^{2}\left[1-\left(\delta / n_{\mathrm{tot}} \Gamma_{m}\right) \cot \theta\right]}{1+4 \eta C n_{\mathrm{tot}}\left(\Gamma_{m} \sin \theta / \delta\right)^{2}\left[1+\left(\delta / n_{\mathrm{tot}} \Gamma_{m}\right) \cot \theta\right]}$

Note that quantum correlations render $R_{\theta}$ antisymmetric in $\theta$ about the amplitude quadrature $\left(\theta=0^{\circ}\right)$; i.e.,

$$
R_{\theta}-1 \approx-\left(R_{-\theta}-1\right) .
$$

It is thus a robust experimental signature for the presence of quantum correlations, provided that excess amplitude and phase noise of the meter laser is sufficiently small (see SM [33] and Ref. [20]). $R_{\theta}$ is measured by recording the spectral power in the windows of the finite bandwidth symmetric about the resonance ( $\delta= \pm 2 \pi \times 21 \mathrm{kHz}$; also see SM [33]), as a function of the homodyne angle $\theta$. Figure 3 shows $R_{\theta}$ for several probe powers. At low probe powers (i.e., low cooperativity, $C \approx 100$ ), shown in the top panel of Fig. 3, the antisymmetric feature around the amplitude quadrature (i.e., $R_{\theta}-1$ ) is relatively small due

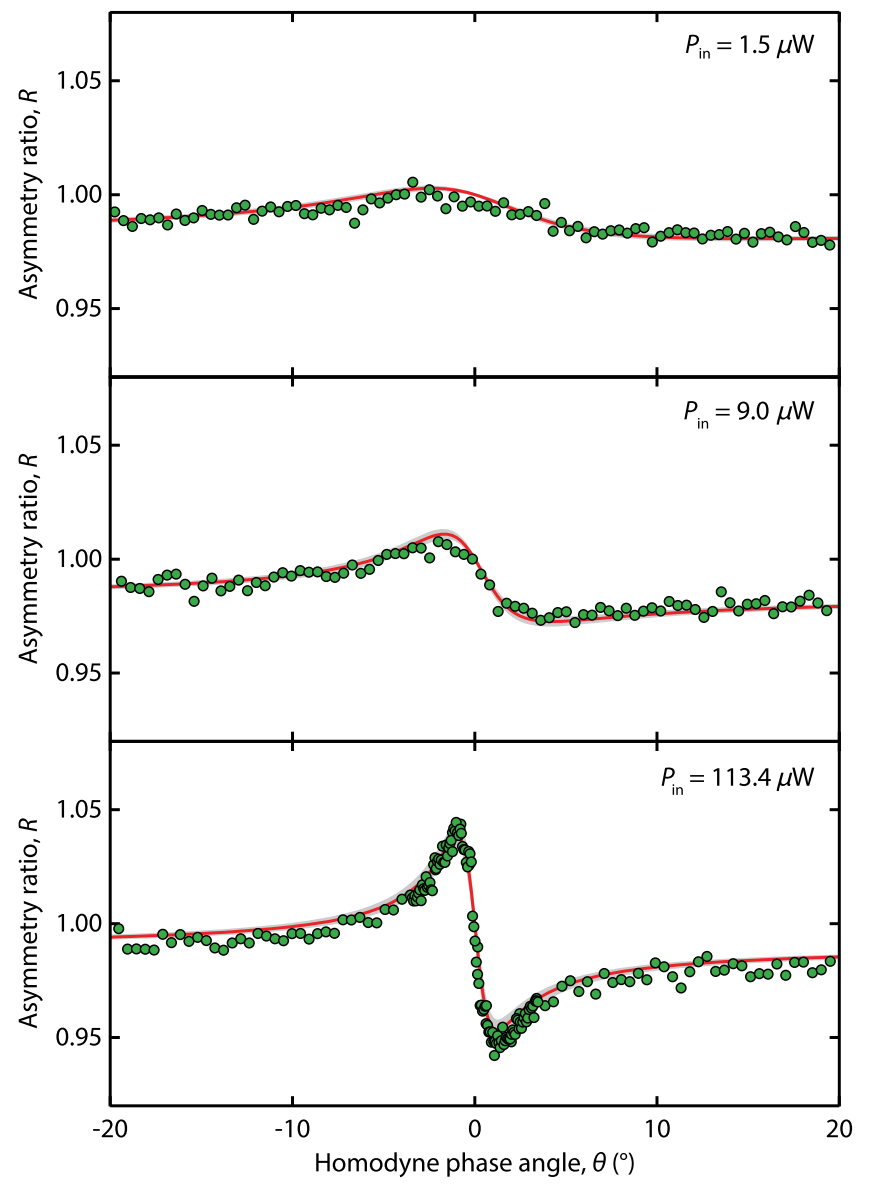

FIG. 3. Asymmetry in spectrum as a function of homodyne angle. Each plot shows asymmetry of the homodyne spectra, $R_{\theta}$ [Eq. (14)], as a function of homodyne angle. From top to bottom, $R_{\theta}$ is plotted as the probe power (mean intracavity photon number) is increased: $P_{\text {in }}=1.5,9,113.4 \mu \mathrm{W}\left(n_{c} \approx 0.3 \times 10^{4}\right.$, $\left.2.1 \times 10^{4}, 58 \times 10^{4}\right)$. Red lines are a model employing only quantum noises and independently inferred values of the effective single-photon cooperativity $\eta C_{0}$; gray band shows interval corresponding to uncertainties in either parameter.

to the large measurement imprecision. As the probe power is increased, shown in the two subsequent panels of Fig. 3, the relative contribution of the quantum correlation increases, leading to a progressively larger antisymmetry near the amplitude quadrature. We note that classical sources of noise may also affect the antisymmetric feature: laser amplitude noise can establish classical amplitudephase correlations leading to excess antisymmetry [38], or anharmonicity of the mechanical oscillator can lead to structured thermal noise which at large Fourier frequency detuning modifies the antisymmetry [40]. These and various other sources of systematics are found to be negligible in our experiment (see SM [33]).

For the scenario in our experiments, where the backaction is large but does not overwhelm thermal motion, i.e., $n_{\text {th }} \gg n_{\text {QBA }} \gg 1$, the visibility of the antisymmetric feature in $R_{\theta \approx 0}$ is given by 


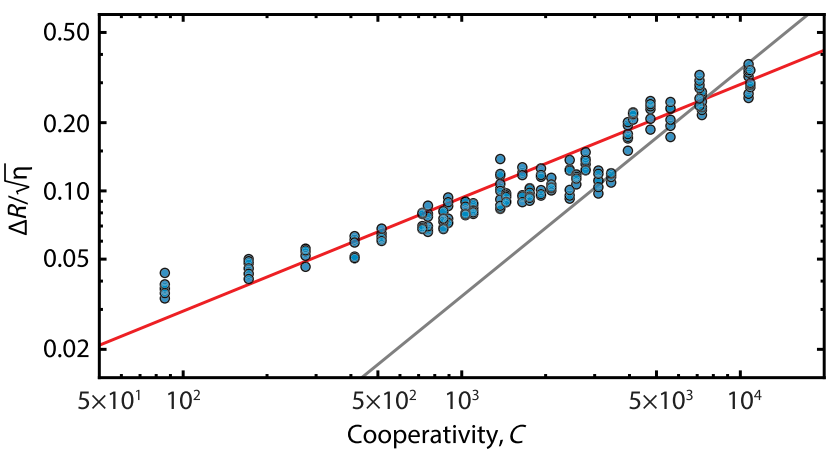

FIG. 4. Visibility of quantum correlations versus laser power. Blue points are measurement of $\Delta R$ [Eq. (16)] as a function of laser power (referred to optomechanical cooperativity $C$ ). At each value of the injected power, the various blue points show $\Delta R$ inferred from various choices of the detuning $\delta$. The red line is a prediction based on Eq. (16), with a notably square-root dependence on power. The gray line is a linear fit to the data, which would apply if the correlations were entirely due to classical amplitude noise in the laser [see Eq. (17)].

$$
\Delta R \equiv \max R_{\theta}-\min R_{\theta} \approx 4 \sqrt{\eta \frac{n_{\mathrm{QBA}}}{n_{\mathrm{th}}}}
$$

Here, the extrema are calculated with respect to both the readout angle $\theta$ and for detuning, $\delta \in\left(\Gamma_{m}, 2 \Gamma_{m} \sqrt{\eta C n_{\mathrm{th}}}\right)$ (see SM [33]). The square-root scaling of $\Delta R$ is unique to quantum correlations (as opposed to correlations produced by classical noise; see SM [33]), and makes possible the $10 \%$ asymmetry despite the relatively small magnitude of quantum backaction in our room-temperature experiment, $n_{\mathrm{QBA}} / n_{\mathrm{th}} \approx 10^{-2}$. In Fig. 4 , we show measurements of $\Delta R$ versus power by analyzing a series of quadrature sweeps as shown in Fig. 3. For all data, $\Delta R$ is extracted from the asymmetry in the same spectral window around $|\delta| \approx$ $2 \pi \times 2 \times 10^{3} \Gamma_{m}$. The observed scaling agrees well with the square-root scaling predicted by Eq. (16), shown as a red line in Fig. 4 with parameters for $C, \eta$, and $n_{\text {th }}$ determined independently.

For comparison, it can be shown that for a laser with excess classical amplitude noise, characterized by an average thermal photon occupation $C_{q q}$ in excess of shot noise, the visibility of the antisymmetric feature is given by (see SM [33])

$$
\Delta R=4 \sqrt{\eta \frac{n_{\mathrm{QBA}}}{n_{\mathrm{th}}}\left(1+2 C_{q q}\right)} .
$$

Phenomenologically, when optical power is changed by attenuating the laser beam (as is done in our experiment), excess amplitude noise scales as $C_{q q} \propto P$ (see SM [33]), leading to $\Delta R \propto P$. This linear scaling is in qualitative disagreement with the observations in Fig. 4.

\section{QUANTUM-NOISE CANCELLATION FOR FORCE SENSING}

Quantum correlations are a generic resource for enhancing the precision with which parameters of a system can be estimated [41]. In the context of interferometric force measurement, two techniques-injection of external correlations $[4,9,11]$ and backaction evasion $[3,42,43]$ — have been conventionally employed to surpass limits imposed by quantum noise of the optical field. A third alternativecorrelations developed in situ-can be directly used to derive a metrological advantage [8,9]. For example, by performing a rotated-quadrature measurement of the cavity output field, an off-resonant external force $\left(F_{\text {ext }}\right)$ applied to the mechanical oscillator, can be estimated with a precision better than that achievable with a phase quadrature measurement. As inferred from the homodyne photocurrent [Eq. (11)], the apparent force experienced by the oscillator (i.e., the force estimator $F_{\text {est }}^{\theta}$ ) has a spectral density (see SM [33])

$$
\begin{aligned}
\bar{S}_{F F}^{\mathrm{est}, \theta}[\Omega]= & \bar{S}_{F F}^{\mathrm{ext}}[\Omega]+\bar{S}_{F F}^{\mathrm{th}}[\Omega] \\
& +\bar{S}_{F F}^{\mathrm{imp}, \theta}[\Omega]+\bar{S}_{F F}^{\mathrm{QBA}}[\Omega]+\hbar \cot \theta \frac{\operatorname{Re} \chi_{x}[\Omega]}{\left|\chi_{x}[\Omega]\right|^{2}},
\end{aligned}
$$

where we now employ the conventional susceptibility, $\chi_{x}[\Omega] \equiv\left[m\left(\Omega_{m}^{2}-\Omega^{2}-i \Omega \Gamma_{m}\right)\right]^{-1}$. Equation (18) shows that the uncertainty in the estimate of $F_{\text {ext }}$ has a classical component due to thermomechanical noise $\left(\bar{S}_{F F}^{\text {th }}\right)$ and a quantum component [second line in Eq. (18)] due to phase quantum noise (imprecision), amplitude shot noise (backaction), and correlations between the two. By detecting a rotated field quadrature $\left(\theta \neq 90^{\circ}\right)$, phase-amplitude correlations $(\alpha \cot \theta)$ can be used to reduce the uncertainty due to quantum noise, which has to be weighed against a concomitant reduction of the signal. In the limit of strong backaction, $\bar{S}_{F F}^{\mathrm{QBA}} \gg \bar{S}_{F F}^{\mathrm{imp}, \pi / 2}$ (i.e., $C \gg 1$ ), and at an optimal measurement quadrature at angle $\theta_{\mathrm{opt}}$, this trade-off reduces to the simplified form (see SM [33]):

$$
\begin{aligned}
\bar{S}_{F F}^{\mathrm{est}, \theta_{\mathrm{opt}}}[\Omega]= & \bar{S}_{F F}^{\mathrm{ext}}[\Omega]+\bar{S}_{F F}^{\mathrm{th}}[\Omega] \\
& +\bar{S}_{F F}^{\mathrm{imp}, \pi / 2}[\Omega]+\left[1-\eta\left(\frac{\operatorname{Re} \chi_{x}[\Omega]}{\left|\chi_{x}[\Omega]\right|}\right)^{2}\right] \bar{S}_{F F}^{\mathrm{QBA}}[\Omega] .
\end{aligned}
$$

Equation (19) shows that measurement backaction can be "erased" from the measured photocurrent at frequencies offset from mechanical resonance. The efficacy of this backaction erasure is limited by the detection efficiency $\eta$. Note that, by contrast, physical backaction is suppressed in backaction evasion schemes [3]. 


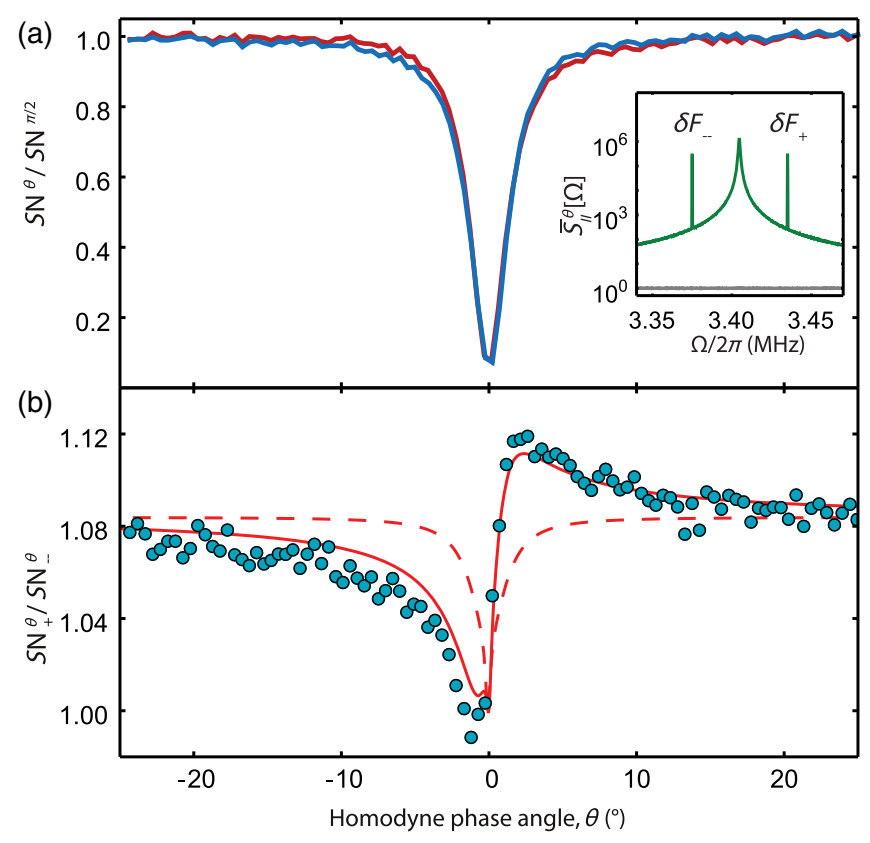

FIG. 5. Quantum-enhanced force estimation. (a) Inset: Green shows homodyne photocurrent spectrum near phase quadrature showing the two balanced forces applied on the mechanical oscillator via radiation pressure from the auxiliary laser; gray is the shot-noise background. Main: Red line (blue line) shows the signal-to-noise ratio of the force $F_{+}\left(F_{-}\right)$normalized to its value for phase quadrature detection, i.e., $\mathrm{SN}_{+(-)}^{\theta} / \mathrm{SN}_{+(-)}^{\pi / 2}$. The asymmetry between the two traces at $\theta \approx \pm 10^{\circ}$ is due to quantumnoise cancellation. Here, the laser input power is $P_{\text {in }}=100 \mu \mathrm{W}$. (b) Blue shows $\mathrm{SN}_{+}^{\theta} / \mathrm{SN}_{-}^{\theta}$, extracted as a ratio of the blue and red traces in (a). For a force balanced in intensity and frequency offset from resonance, Eq. (22) predicts that $\mathrm{SN}_{+}^{\theta} / \mathrm{SN}_{-}^{\theta} \propto R_{\theta}$. The solid red line is a prediction based on Eq. (22) in conjunction with Eq. (14), while red dashed line shows the same model excluding the contribution from quantum correlations.

In the presence of thermal noise, the signal-to-noise enhancement afforded by quantum correlations in Eq. (19) is diminished by a factor of approximately $n_{\mathrm{th}} / n_{\mathrm{QBA}}$ (see SM [33]). We nevertheless observe the principle behind this quantum enhancement by applying a detuned radiation force $F_{\text {ext }}\left[\Omega_{\text {ext }}\right]$ via an auxiliary cavity field (the same used for feedback; see SM [33]) and recording the signal-tonoise ratio,

$$
\mathrm{SN}^{\theta}\left[\Omega_{\mathrm{ext}}\right] \equiv \frac{\bar{S}_{F F}^{\mathrm{est}, \theta}\left[\Omega_{\mathrm{ext}}\right]}{\bar{S}_{F F}^{\text {est } \theta}\left[\Omega_{\mathrm{ext}}\right]-\bar{S}_{F F}^{\mathrm{ext}}\left[\Omega_{\mathrm{ext}}\right]},
$$

in the homodyne photocurrent versus $\theta$. In the experiment, we choose a two-tone force of the form

$$
F_{\mathrm{ext}}[\Omega]=F_{+} \delta\left[\Omega_{m}+\delta\right]+F_{-} \delta\left[\Omega_{m}-\delta\right],
$$

centered around resonance, so that one of the forces provides a reference for the signal-to-noise ratio. We denote by $\mathrm{SN}_{ \pm}^{\theta}$ the definition of the signal-to-noise ratio in Eq. (20) applied to each of the two force components $\left(F_{ \pm}\right)$. Figure 5(a) shows the variation of $\mathrm{SN}_{ \pm}^{\theta}$ for each of the forces $F_{ \pm}$(carefully balanced, as shown in inset) as the homodyne readout angle is varied. The effect of quantum correlations in the optical field is to cancel backaction at intermediate measurement quadratures $\left(0^{\circ}<|\theta|<90^{\circ}\right)$, leading to an enhancement or suppression of $\mathrm{SN}_{ \pm}^{\theta}$ at these optimal quadratures. For a fixed measurement setting (i.e., fixed value of $C, \theta, \delta$ ), it can be seen that quantum-noise cancellation leads to an enhanced signal-to-noise ratio for $F_{+}$relative to $F_{-}$, or visa versa. The absolute value of the enhancement is given by the ratio

$$
\frac{\mathrm{SN}^{\theta}\left[\Omega_{m}+\delta\right]}{\mathrm{SN}^{\theta}\left[\Omega_{m}-\delta\right]} \approx \frac{1}{R_{\theta}} \frac{\left|\chi_{x}\left[\Omega_{m}+\delta\right]\right|^{2}}{\left|\chi_{x}\left[\Omega_{m}-\delta\right]\right|^{2}} \frac{\left\langle F_{+}^{2}\right\rangle}{\left\langle F_{-}^{2}\right\rangle}
$$

This ratio is plotted in Fig. 5(b). The observed antisymmetric dependence on $\theta$ is directly related to $R_{\theta}$ [Eq. (14)]. The offset from unity at $\theta= \pm 90^{\circ}$ is due to imperfect balance of the two forces. The maximum deviation (at $\theta \approx \pm 2^{\circ}$ ) of approximately $12 \%$ is less than the absolute signal loss shown in Fig. 5(a). It nevertheless provides a measure of the improvement obtained in the ability to estimate a force with and without quantum-noise cancellation.

\section{CONCLUSION}

To summarize, we demonstrate a room-temperature cavity-enhanced interferometer where quantum correlations of light are generated in situ via the radiation pressure interaction between light and the effective harmonic motion of the cavity. We show that these correlations manifest as a $10 \%$ cancellation of quantum noise in a homodyne measurement away from phase quadrature - variational measurement - of the optical field over an octave in frequency about $3 \mathrm{MHz}$ from the optical carrier. The absolute magnitude of quantum-noise cancellation is shown to scale as the square root of the ratio of the backaction to thermal force acting on the mechanical oscillator. Finally, by applying an external force to the oscillator, we demonstrate how quantum-noise cancellation leads to an increase in the signal-to-noise ratio for the external force. In future experiments, where backaction is the dominant force noise, the "variational" measurement technique, applied here at room temperature, can be used to surpass the SQL for a linear force measurement. This remains a long-standing pursuit in the gravitational wave community, and was recently demonstrated in a microcavity optomechanical system at dilution refrigerator temperatures to obtain a displacement sensitivity beyond the finiteefficiency standard quantum limit [44].

Data and data analysis code used for this work is available at [45]. 


\section{ACKNOWLEDGMENTS}

All samples are fabricated at the Center for MicroNanoTechnology (CMi) at EPFL. This work was supported by funding from the European Union's Horizon 2020 research program through a Marie Sklodowska-Curie ETN grant (HOT), an ERC Advanced Grant (QuREM), the Swiss National Science Foundation's grant for Cavity Quantum Optomechanics, and the NCCR for Quantum Engineering (QSIT).

V.S., R.S., S. A.F., and H.S. contributed equally to this work.

[1] V. B. Braginsky and A. B. Manukin, Ponderomotive Effects of Electromagnetic Radiation, Sov. Phys. JETP 25, 653 (1967).

[2] C. M. Caves, Quantum-Mechanical Radiation-Pressure Fluctuations in an Interferometer, Phys. Rev. Lett. 45, 75 (1980).

[3] C. M. Caves, M. Zimmermann, K. S. Thorne, and R. W. Drever, On the Measurement of a Weak Classical Force Coupled to a Quantum-Mechanical Oscillator. I. Issues of Principle, Rev. Mod. Phys. 52, 341 (1980).

[4] M. T. Jaekel and S. Reynaud, Quantum Limits in Interferometric Measurements, Europhys. Lett. 13, 301 (1990).

[5] C. Fabre, M. Pinard, S. Bourzeix, A. Heidmann, E. Giacobino, and S. Reynaud, Quantum-Noise Reduction Using a Cavity with a Movable Mirror, Phys. Rev. A 49, 1337 (1994).

[6] S. Mancini and P. Tombesi, Quantum Noise Reduction by Radiation Pressure, Phys. Rev. A 49, 4055 (1994).

[7] S. P. Vyatchanin and E. A. Zubova, Quantum Variation Measurement of a Force, Phys. Lett. A 201, 269 (1995).

[8] A. Buonanno and Y. Chen, Quantum Noise in Second Generation, Signal-Recycled Laser Interferometric Gravitational-Wave Detectors, Phys. Rev. D 64, 042006 (2001).

[9] H. J. Kimble, Y. Levin, A. B. Matsko, K. S. Thorne, and S. P. Vyatchanin, Conversion of Conventional Gravitational-Wave Interferometers into Quantum Nondemolition Interferometers by Modifying Their Input and/or Output Optics, Phys. Rev. D 65, 022002 (2001).

[10] Y. Chen, S. L. Danilishin, F. Ya Khalili, and H. MüllerEbhardt, QND Measurements for Future GravitationalWave Detectors, Gen. Relativ. Gravit. 43, 671 (2011).

[11] J. Aasi et al., Enhanced Sensitivity of the LIGO Gravitational Wave Detector by Using Squeezed States of Light, Nat. Photonics 7, 613 (2013).

[12] H. Vahlbruch, M. Mehmet, K. Danzmann, and R. Schnabel, Detection of $15 d B$ Squeezed States of Light and Their Application for the Absolute Calibration of Photoelectric Quantum Efficiency, Phys. Rev. Lett. 117, 110801 (2016).

[13] M. Aspelmeyer, T. J. Kippenberg, and F. Marquardt, Cavity Optomechanics, Rev. Mod. Phys. 86, 1391 (2014).

[14] T. P. Purdy, R. W. Peterson, and C. A. Regal, Observation of Radiation Pressure Shot Noise on a Macroscopic Object, Science 339, 801 (2013).
[15] D. J. Wilson, Vivishek Sudhir, N. Piro, R. Schilling, A. Ghadimi, and T. J. Kippenberg, Measurement-Based Control of a Mechanical Oscillator at Its Thermal Decoherence Rate, Nature (London) 524, 325 (2015).

[16] J. D. Teufel, F. Lecocq, and R. W. Simmonds, Overwhelming Thermomechanical Motion with Microwave Radiation Pressure Shot Noise, Phys. Rev. Lett. 116, 013602 (2016).

[17] D. W. C. Brooks, T. Botter, S. Schreppler, T. P. Purdy, N. Brahms, and D. M. Stamper-Kurn, Non-Classical Light Generated by Quantum-Noise-Driven Cavity Optomechanics, Nature (London) 488, 476 (2012).

[18] A. H. Safavi-Naeini, S. Gröblacher, J. T. Hill, J. Chan, M. Aspelmeyer, and O. Painter, Squeezed Light from a Silicon Micromechanical Resonator, Nature (London) 500, 185 (2013).

[19] T. P. Purdy, P. Yu, R. W. Peterson, N. S. Kampel, and C. A. Regal, Strong Optomechanical Squeezing of Light, Phys. Rev. X 3, 031012 (2013).

[20] V. Sudhir, D. J. Wilson, R. Schilling, H. Schütz, S. A. Fedorov, A. H. Ghadimi, A. Nunnenkamp, and T. J. Kippenberg, Appearance and Disappearance of Quantum Correlations in Measurement-Based Feedback Control of a Mechanical Oscillator, Phys. Rev. X 7, 011001 (2017).

[21] W. H. P. Nielsen, Y. Tsaturyan, C. Bo Møller, E. S. Polzik, and A. Schliesser, Multimode Optomechanical System in the Quantum Regime, Proc. Natl. Acad. Sci. U.S.A. 114, 62 (2017).

[22] F. Ya. Khalili, H. Miao, H. Yang, A. H. Safavi-Naeini, O. Painter, and Y. Chen, Quantum Back-Action in Measurements of Zero-Point Mechanical Oscillations, Phys. Rev. A 86, 033840 (2012).

[23] A. J. Weinstein, C. U. Lei, E. E. Wollman, J. Suh, A. Metelmann, A. A. Clerk, and K. C. Schwab, Observation and Interpretation of Motional Sideband Asymmetry in a Quantum Electromechanical Device, Phys. Rev. X 4, 041003 (2014).

[24] T. P. Purdy, P.-L. Yu, N. S. Kampel, R. W. Peterson, K. Cicak, R. W. Simmonds, and C. A. Regal, Optomechanical Raman-Ratio Thermometry, Phys. Rev. A 92, 031802 (2015).

[25] M. Underwood, D. Mason, D. Lee, H. Xu, L. Jiang, A. B. Shkarin, K. Børkje, S. M. Girvin, and J. G. E. Harris, Measurement of the Motional Sidebands of a NanogramScale Oscillator in the Quantum Regime, Phys. Rev. A 92, 061801 (2015).

[26] M. Evans et al., Observation of Parametric Instability in Advanced LIGO, Phys. Rev. Lett. 114, 161102 (2015).

[27] P. Verlot, A. Tavernarakis, T. Briant, P.-F. Cohadon, and A. Heidmann, Backaction Amplification and Quantum Limits in Optomechanical Measurements, Phys. Rev. Lett. 104, 133602 (2010).

[28] K. Borkje, A. Nunnenkamp, B. Zwickl, C. Yang, J. Harris, and S. Girvin, Observability of Radiation-Pressure Shot Noise in Optomechanical Systems, Phys. Rev. A 82, 013818 (2010).

[29] T. P. Purdy, K. E. Grutter, K. Srinivasan, and J. M. Taylor, Quantum Correlations from a Room-Temperature Optomechanical Cavity, Science 356, 1265 (2017). 
[30] V. B. Braginsky, M. L. Gorodetsky, F. Ya. Khalili, and K. S. Thorne, Dual-Resonator Speed Meter for a Free Test Mass, Phys. Rev. D 61, 044002 (2000).

[31] T. Corbitt, Y. Chen, F. Khalili, D. Ottaway, S. Vyatchanin, S. Whitcomb, and N. Mavalvala, Squeezed-State Source Using Radiation-Pressure-Induced Rigidity, Phys. Rev. A 73, 023801 (2006).

[32] R. Schilling, H. Schütz, A. H. Ghadimi, V. Sudhir, D. J. Wilson, and T. J. Kippenberg, Near-Field Integration of a SiN Nanobeam and a $\mathrm{SiO}_{2}$ Microcavity for HeisenbergLimited Displacement Sensing, Phys. Rev. Applied 5, 054019 (2016).

[33] See Supplemental Material at http://link.aps.org/ supplemental/10.1103/PhysRevX.7.031055 for an extensive theoretical discussion and additional details of the experiment.

[34] Here, we adopt the definition, $\delta q_{\text {in }}=\left(\delta a_{\text {in }}+\delta a_{\text {in }}^{\dagger}\right) / \sqrt{2}$, for the amplitude quadrature. This, in conjunction with the conventional correlator for the photon flux, gives a factor of $1 / 2$ in the correlator for the amplitude quadrature.

[35] L. F. Buchmann, S. Schreppler, J. Kohler, N. Spethmann, and D. M. Stamper-Kurn, Complex Squeezing and Force Measurement Beyond the Standard Quantum Limit, Phys. Rev. Lett. 117, 030801 (2016).

[36] J. B. Clark, F. Lecocq, R. W. Simmonds, J. Aumentado, and J. D. Teufel, Observation of Strong Radiation Pressure Forces from Squeezed Light on a Mechanical Oscillator, Nat. Phys. 12, 683 (2016).

[37] T. Caniard, P. Verlot, T. Briant, P.-F. Cohadon, and A. Heidmann, Observation of Back-Action Noise Cancellation in Interferometric and Weak Force Measurements, Phys. Rev. Lett. 99, 110801 (2007).

[38] F. Marino, F. S. Cataliotti, A. Farsi, M. S. de Cumis, and F. Marin, Classical Signature of Ponderomotive Squeezing in a Suspended Mirror Resonator, Phys. Rev. Lett. 104, 073601 (2010).

[39] P. F. Cohadon, A. Heidmann, and M. Pinard, Cooling of a Mirror by Radiation Pressure, Phys. Rev. Lett. 83, 3174 (1999).

[40] S. A. Fedorov, V. Sudhir, R. Schilling, H. Schütz, D. J. Wilson, and T.J. Kippenberg, Evidence for Structural Damping in a High-Stress Silicon Nitride Nanobeam and Its Implications for Quantum Optomechanics, Phys. Lett. A, DOI: 10.1016/j.physleta.2017.05.046 (2017).

[41] V. Giovannetti, S. Lloyd, and L. Maccone, QuantumEnhanced Measurements: Beating the Standard Quantum Limit, Science 306, 1330 (2004).

[42] J. Suh, A. J. Weinstein, C. U. Lei, E. E. Wollman, S. K. Steinke, P. Meystre, A. A. Clerk, and K. C. Schwab, Mechanically Detecting and Avoiding the Quantum Fluctuations of a Microwave Field, Science 344, 1262 (2014).

[43] F. Lecocq, J. B. Clark, R. W. Simmonds, J. Aumentado, and J. D. Teufel, Quantum Nondemolition Measurement of a Nonclassical State of a Massive Object, Phys. Rev. X 5, 041037 (2015).

[44] N. S. Kampel, R. W. Peterson, R. Fischer, P.-L. Yu, K. Cicak, R. W. Simmonds, K. W. Lehnert, and C. A. Regal, Improving Broadband Displacement Detection with Quantum Correlations, Phys. Rev. X 7, 021008 (2017).

[45] http://dx.doi.org/10.5281/zenodo.854557. 